\title{
The EEG in psychiatry
}

\author{
Roland Eastman \\ Division of Neurology, University of Cape Town, Cape Town, South Africa
}

The value of EEG in the practice of psychiatry has been a debated issue since the advent of these neurophysiological studies in the 1930's. Surprisingly, there are few credible studies in this area, and much of the earlier work is bedevilled by poor research design and hence unwarranted conclusions. A brief review in this issue highlights some of the conflicting reports and ventures the opinion that routine referral of all patients attending a psychiatric service is not appropriate in view of the likely low yield of results which will change the patient's management, and the attendant risk of over-interpretation of minor non-specific findings which may lead to falsepositive diagnoses.

I would agree with the view expressed that we do not at present have a sufficient base of evidence from which to form firm guidelines, and that this information is sorely needed. Nevertheless, we need to proceed with what we have, and, in my opinion, there is a clear contribution that EEG may at times make in attempting to diagnose the symptoms encountered in psychiatry. In everyday clinical work, the EEG remains the only practical functional test of brain function, and, as such, complements the fine anatomical and pathological detail given by modern imaging. The value of an EEG depends heavily upon the diagnosis. It is especially of great value in assisting with the identification of epilepsy and of organic mental dis-

\author{
Correspondence: \\ Professor R Eastman \\ Division of Neurology, Groote Schuur Hospital, University of Cape \\ Town, Observatory, Cape Town, South Africa \\ email:reastman@uctgsh1.uct.ac.za
}

orders. Epilepsy is primarily a clinical diagnosis, but the EEG may provide strong support by the finding of inter-ictal epileptogenic discharges and also be used to define the site of seizure onset and the epilepsy syndrome. However, a normal inter-ictal EEG can never refute or exclude a clinical diagnosis of epilepsy. Organic mental disorders is increasingly an unsatisfactory term, as many of the so-called functional psychiatric disorders have a neurobiological basis. Nevertheless, the typically marked focal or generalised slowing found in the EEG in patients with acute or chronic encephalopathies due to metabolic changes, infections, toxins, trauma and tumours is useful to the clinician in the differentiation of these disorders from psychiatric disorders. However, a normal EEG does not exclude all forms of structural disease, and in particular extra-parenchymal intra-cranial lesions such as meningiomata or subdural haematomas typically result in no EEG abnormalities.

Turning to psychiatric disorders, it is clear that it is not uncommon to find typically minor EEG abnormalities in some syndromes and this is not surprising. Perhaps the best recognised is the non-specific EEG slowing often found in the temporal and central areas in aggressive psychopaths.

It becomes clear then that the frequency and clinical value of EEG abnormalities found in psychiatric patients depends very much upon the case-mix, and the differential diagnosis being considered. And it is not sufficient to regard merely the EEG abnormalities as being of potential use, as it may be just as worthwhile to know that the study is normal. As always the clinical value of a test depends upon the question being asked, and the likelihood of finding an answer.

As suggested a rigorous study under local conditions would indeed be of interest.

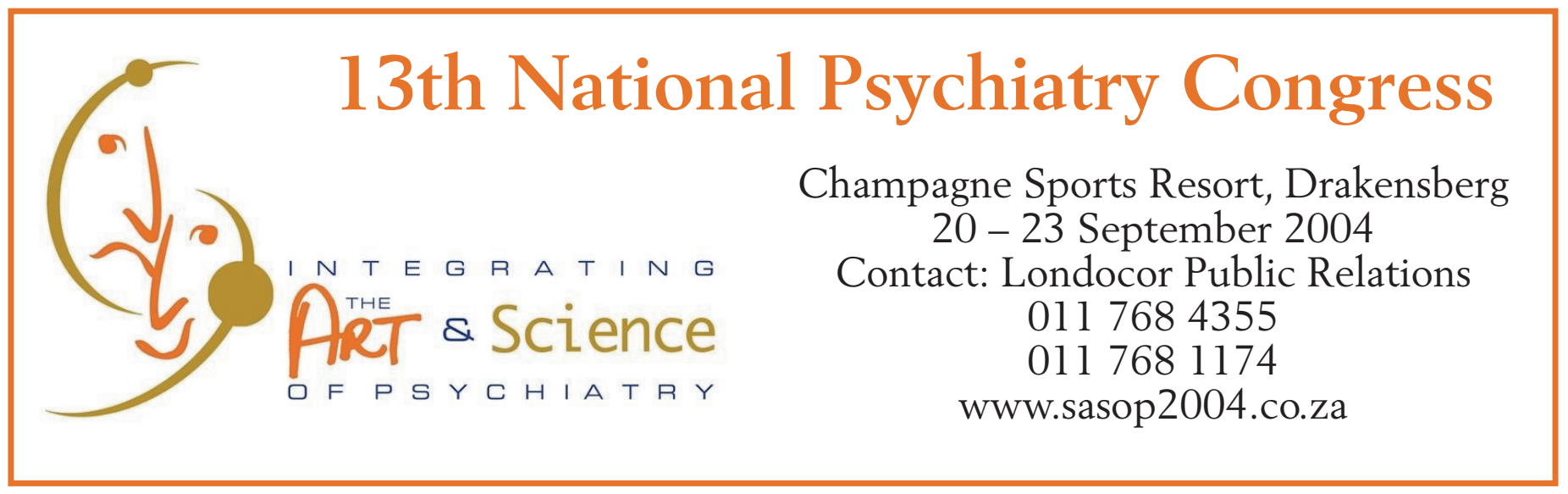

